\title{
Les produits de l'anacardier : caractéristiques, voies de valorisation et marchés
}

Emmanuelle Lautiéa, b, Manuel Dornier ${ }^{a, b *}$, M. de Souza Filhoc, Max Reynes ${ }^{a, b}$

\author{
a École nationale supérieure \\ des Industries alimentaires, \\ section Industries alimentaires \\ régions chaudes (Ensia-Siarc), \\ BP 5098, \\ 34033 Montpellier Cedex 01, \\ France \\ b Centre de coopération \\ international en recherche \\ agronomique \\ pour le développement \\ (Cirad-flhor), \\ TA 50 / PS4, bld de la Lironde, \\ 34398 Montpellier Cedex 05, \\ France \\ ${ }^{c}$ Empresa Brasileira \\ de Pesquisa Agropecuaria \\ (Embrapa), \\ Rua Dra Sara Mesquita 2270 \\ Planalto Pici, C.P. 3761 , \\ 60511-110 Fortaleza-Ceara, \\ Brésil
}

dornier@cirad.fr

* Correspondence and reprints

Reçu le 23 octobre 2000 Accepté le 9 mars 2001

Fruits, 2001, vol. 56, p. 235-248 (C) $2001 \mathrm{Cirad} /$ EDP Sciences All rights reserved

RESUMEN ESPAÑoL, p. 248

\section{The cashew tree and its products: main characteristics and possible uses.}

Abstract - Introduction. Indigenous to Brazil, the cashew tree is present in many countries of the intertropical area. This work is a summing up of the economic interests and the different uses of such a widespread tree. The cashew tree. Anacardium occidentale (Anacardiacea) is a rather resistant species, especially resistant to the dry periods, which makes it a tree welladapted to many tropical areas. In spite of its tolerance to parasitic attack, it can be susceptible to several insects and some fungi attacks. The average yields obtained for nuts are approximately $600 \mathrm{~kg} \times \mathrm{ha}^{-1} \times$ year- $^{-1}$ and they are likely to be improved thanks to the varietal choice. The cashew nut. Biologically, it is the real fruit of the plant. The almond has a high content of lipids and has got approximately $20 \%$ of proteins. In 1999, the main world producers, each with a production higher than 100,000 t, were India, Nigeria, Brazil and Tanzania. After the crushing of the hull and the pruning process, this product is generally exported. The cashew apple. With maturity, the hypertrophied stalk of the nut turns into a sweet, fleshy and juicy fruit. It is remarkable for its astringency and its high vitamin C content $\left(200-300 \mathrm{mg} \times 100 \mathrm{~g}^{-1}\right)$. The cashew apple is used rather little except in India and in Brazil, as fruit juice for example. Other productions of the cashew tree. The cashew nut shell liquid (CNSL) is a rather original secondary production: extracted from the hulls of nuts, this corrosive liquid rich in phenolic compounds is used in the chemical industry. Conclusion. Cashew farming with its diversity of products seems to be able to constitute an interesting source of incomes for the dry tropical areas, especially with the nut which is well integrated into the international markets. Nevertheless, the apple requires better development in many places because it is presently a by-product of the cashew nut with a strong potential.

Anacardium occidentale L. / cashew / chemical composition / processing / commodity markets / world markets / uses / fruit juices / tannins

\section{Les produits de l'anacardier : caractéristiques, voies de valorisation et marchés.}

Résumé - Introduction. Originaire du Brésil, l'anacardier est largement présent dans la zone intertropicale. Une synthèse sur les intérêts économiques et les différentes voies de valorisations des produits de cet arbre a été effectuée. L'anacardier. De part sa résistance, notamment aux périodes de sécheresse, l'espèce Anacardium occidentale (anacardiacée) est adaptée à de nombreuses zones tropicales. Malgré sa tolérance aux attaques parasitaires, elle peut être sensible à certains insectes et attaques fongiques comme l'oïdium. Les rendements moyens obtenus pour la noix sont d'environ $600 \mathrm{~kg} \times \mathrm{ha}^{-1} \times \mathrm{an}^{-1}$; ils sont susceptibles d'être améliorés grâce à la sélection variétale. La noix de cajou. Elle est le fruit de l'anacardier au sens botanique du terme. L'amande est riche en lipides et contient environ $20 \%$ de protéines. En 1999, les principaux producteurs mondiaux de noix de cajou ont été l'Inde, le Nigéria, le Brésil et la Tanzanie, qui ont produit chacun plus de $100000 \mathrm{t} \times \mathrm{an}^{-1}$. Après concassage de la coque et émondage, ce produit à forte valeur ajoutée est le plus souvent exporté. La pomme de cajou. Elle correspond au pédoncule hypertrophié de la noix qui possède à maturité une chair juteuse et sucrée. Ses principales caractéristiques sont son astringence et sa teneur élevée en vitamine $C\left(200-300 \mathrm{mg} \times 100 \mathrm{~g}^{-1}\right)$. La pomme de cajou est assez peu valorisée sauf en Inde et au Brésil, notamment sous forme de jus de fruit. Autres productions. Le baume de cajou est une production secondaire assez originale : extrait des coques de la noix, ce liquide corrosif, riche en composés phénoliques, est utilisé dans l'industrie chimique. Conclusion. La culture de l'anacardier, avec sa diversité de produits et notamment avec la noix qui est bien intégrée aux marchés internationaux, semble pouvoir constituer une source intéressante de revenus pour les zones tropicales sèches. Néanmoins, la pomme mériterait d'être mieux valorisée car elle reste encore un sous-produit de l'industrie de la noix de cajou.

Anacardium occidentale L. / noix de cajou / composition chimique / traitement / marché des produits de base / marché mondial / utilisation / jus de fruits / tannin 


\section{Introduction}

L'anacardier serait originaire du nord-est brésilien; le nom «cajou »pourrait dériver du tupi guarani (tribu indienne du Nord-Est du Brésil) «acâ-yú » qui signifie «pomme jaune »[1].

La dissémination de l'anacardier au sein du continent sud-américain a été spontanée et naturelle alors que sa présence dans les autres continents est attribuée à l'intervention de l'homme. L'arbre fut introduit par les Portugais au Mozambique et en Inde entre 1563 et 1578 [2], puis dans le Sud-Est asiatique. L'anacardier était alors surtout cultivé pour la lutte contre l'érosion des sols et la protection des cultures plus fragiles grâce à la densité de son feuillage. Il s'est répandu en Afrique durant la deuxième moitié du XVIe siècle, d'abord sur la côte est, puis vers l'ouest. Alors que l'introduction de l'espèce sur les continents africain et asiatique est attribuée à l'homme, il semblerait que le principal vecteur de son extension ait été l'éléphant par le fait de la dissémination, à travers toute la côte estafricaine, des noix peu digestes ingérées en même temps que les pommes de cajou. L'arbre s'est ensuite répandu en Australie et dans certaines zones du continent nordaméricain, par exemple en Floride. Aujourd'hui, l'anacardier se rencontre pratiquement partout entre $31^{\circ}$ de latitude nord et $31^{\circ}$ de latitude sud sous des dénominations très variables (tableau I).

Compte tenu de l'importance économique actuelle de l'anacardier, nous avons entrepris de faire une synthèse des caractéristiques de l'arbre ainsi que des utilisations actuelles et potentielles de ses productions.

\section{L'anacardier}

\subsection{Caractéristiques agronomiques}

L'anacardier, Anacardium occidentale L., appartient à la famille des anacardiacées dont font également partie le manguier (Mangifera indica L.) et le pistachier (Pistacia vera L.). C'est un arbre d'une dizaine de mètres, possédant un feuillage dense et ramifié, dont le diamètre peut atteindre de 12 à $15 \mathrm{~m}$ [2]. Les feuilles sont alternes, simples et coriaces. Les fleurs, nombreuses et regroupées en panicule terminale [3], sont principalement hermaphrodites même s'il est possible de trouver jusqu'à $20 \%$ de fleurs mâles [2].

\subsubsection{Zones écologiques}

L'anacardier se développe de préférence à des altitudes inférieures à $1000 \mathrm{~m}$, dans des zones au climat tropical chaud, avec une alternance de saisons sèches et humides. Il s'adapte à des régimes pluviométriques divers mais, pour une bonne fructification, une pluviométrie annuelle comprise entre 760 et $1800 \mathrm{~mm}$ est préférable [3].

L'anacardier s'adapte à divers types de sols. Il n'exige pas un sol riche mais préfère les sols légers, sableux, profonds, bien drainés et composés à $25 \%$ d'argile. Sur les cuirasses latéritiques et les bas-fonds, il végète car son pivot est sensible aux obstacles indurés et à l'inondation [3]. Les pH favorables varient de 4,5 à 6,5 [4]. Il peut être sensible à des carences en magnésium [5] mais il est reconnu comme un arbre résistant, adapté à de nombreuses zones tropicales, car il est peu sensible à la sécheresse : une saison sèche de 6 mois favorise la fructification et permet une bonne conservation des graines. En revanche, la plante, qui supporte des températures s'échelonnant de 12 à $32{ }^{\circ} \mathrm{C}$, est sensible aux écarts de température ainsi qu'au froid.

\subsubsection{Ravageurs et maladies}

Bien que l'anacardier soit une espèce résistante, connue pour son adaptabilité et sa tolérance aux attaques parasitaires, elle est tout de même sujette à certains insectes et aux attaques fongiques. Les plus répandues sont celles qui causent l'anthracnose, la moisissure noire et l'oïdium, mais on peut citer d'autres phytopathogènes comme Phytophthora palmivora et P. nicotianae, Septoria cajui et Sclerotium rolfsii [2]. Les attaques qui causent le plus de pertes sont celles qui concernent les inflorescences ou les fruits puisqu'elles nuisent directement à la production. 
Tableau I.

Principaux noms vernaculaires de l'anacardier et de ses différents produits [2].

\begin{tabular}{|c|c|c|c|c|c|}
\hline \multirow{2}{*}{$\begin{array}{l}\text { Langue } \\
\text { Français }\end{array}$} & \multicolumn{5}{|c|}{ Différents produits de Anacardium occidentale } \\
\hline & $\begin{array}{l}\text { Cajou } \\
\text { Acajou }\end{array}$ & Anacardier & Noix de cajou & Pomme de cajou & Amande de cajou \\
\hline Portugais & Cajú & $\begin{array}{l}\text { Cajueiro } \\
\text { Pé de cajú }\end{array}$ & Castanha de cajú & $\begin{array}{l}\text { Pedúnculo } \\
\text { Falso-fruto } \\
\text { Maça de cajú }\end{array}$ & $\begin{array}{c}\text { Amêndoa de } \\
\text { Castanha de cajú }\end{array}$ \\
\hline Espagnol & $\begin{array}{l}\text { Anacardo } \\
\text { Marañon } \\
\text { Cajuil }\end{array}$ & $\begin{array}{c}\text { Anacardo } \\
\text { Marañon } \\
\text { Merey } \\
\text { (au Vénézuela) }\end{array}$ & $\begin{array}{l}\text { Nuez de anacardo } \\
\text { Nuez de marañon }\end{array}$ & $\begin{array}{l}\text { Falso fruto } \\
\text { Manzana } \\
\text { de anacardo }\end{array}$ & $\begin{array}{c}\text { Almendra } \\
\text { de marañon }\end{array}$ \\
\hline Anglais & Cashew & Cashew tree & Cashew nut & Cashew apple & Cashew kernel \\
\hline Italien & - & Anacardio & Noce d'anacardio & Mandorla d'anacardio & - \\
\hline Swahili & Mkanju & - & Korosho & - & - \\
\hline Somalien & Bibbo & - & Bibs & - & - \\
\hline Philippin & Kasoy & - & Kasiu & - & - \\
\hline Indonésien & Jambu mente & - & Jambu mete & - & - \\
\hline Thai & Ma-muang & - & Imapan & - & - \\
\hline
\end{tabular}

Les insectes nuisibles les plus couramment rencontrés sont des chenilles dévoreuses et enrouleuses de feuilles (Eutelia et Sylepta), les cochenilles, les thrips, les flatides [3], ainsi que trois espèces d'Helopeltis (H. anacardii, H. shoutedeni et H. antonii [2]) qui piquent les feuilles, les bourgeons et les pousses tendres créant des taches brunes qui se nécrosent en quelques jours. Dans certains pays d'Afrique, un insecte attaquant les cocotiers, Pseudotheraptus wayi, peut aussi affecter l'anacardier [2]. Enfin, Acrocercops syngramma peut aussi causer des pertes dans la production en provoquant la chute des feuilles.

\subsubsection{Fructification}

Selon les conditions climatiques, l'anacardier peut être cultivé pour sa fructification ou pour le reboisement. La durée de vie de l'arbre est d'environ 30 ans. Il produit des fruits à partir de 4 à 5 ans après la germination, ou après 3 ans si la multiplication est végétative [6]. Le fruit (la noix) se développe de 6 à 8 semaines après la pollinisation réalisée par des insectes. Le faux-fruit (la pomme) se développe seulement durant les deux dernières semaines [5].

Les deux types de fruits atteignent leur maturité environ au même moment. La récolte est difficile à mécaniser à cause, notamment, de la hauteur et de la largeur du feuillage, même si une mécanisation partielle est possible en adaptant la technique utilisée pour les oliviers [2]. Les fruits sont donc généralement ramassés manuellement après être tombés au sol. La maturation progressive impose de renouveler la récolte tous les 2 à 4 jours pour les pommes de cajou et tous les 7 à 10 jours si seules les noix sont valorisées.

\subsection{Sélection variétale}

La sélection des variétés est très fréquemment mise en œuvre pour, principalement, améliorer la culture et augmenter la production. Les critères de sélection sont variés : rendement et hauteur des arbres, résistance à certaines maladies comme l'oïdium, dimension du fruit, caractéristiques 
physicochimiques des noix et des pédoncules, etc.

Les différents pays producteurs ont pris conscience que les plantations issues de graines présentaient un certain nombre d'inconvénients : production hétérogène, taux important de plantes atypiques et à faibles rendements [2]. Des programmes de sélection avec intervention génétique ont donc été entrepris en Inde, au Brésil, en Thailande, en Tanzanie et en Chine.

À titre d'exemple, on peut citer le cas du Cearà, État du Nord-Est du Brésil, où des programmes d'amélioration ont commencé dès 1965 [2] ; quatre «clones » (CCP06, CCP09, CCP76 et CCP1001) d'anacardier nain précoce (A. occidentale var. nanum) ont été isolés. Ils mesurent de 4 à $5 \mathrm{~m}$, ce qui facilite considérablement la récolte manuelle, possèdent une durée de fructification plus longue (8 mois), ce qui permet d'étaler la production sur une période plus grande ; leur rendement (1300 kg $\times$ ha $^{-1}$ $\times \mathrm{an}^{-1}$ de noix) est supérieur à celui des autres anacardiers et ils produisent des pommes cajou moins astringentes. De plus, la production peut commencer dès la première année contrairement à l'anacardier commun.

Figure 1.

La noix et la pomme de cajou, vrai fruit et faux fruit de l'anacardier

(Anacardium occidentale L.).

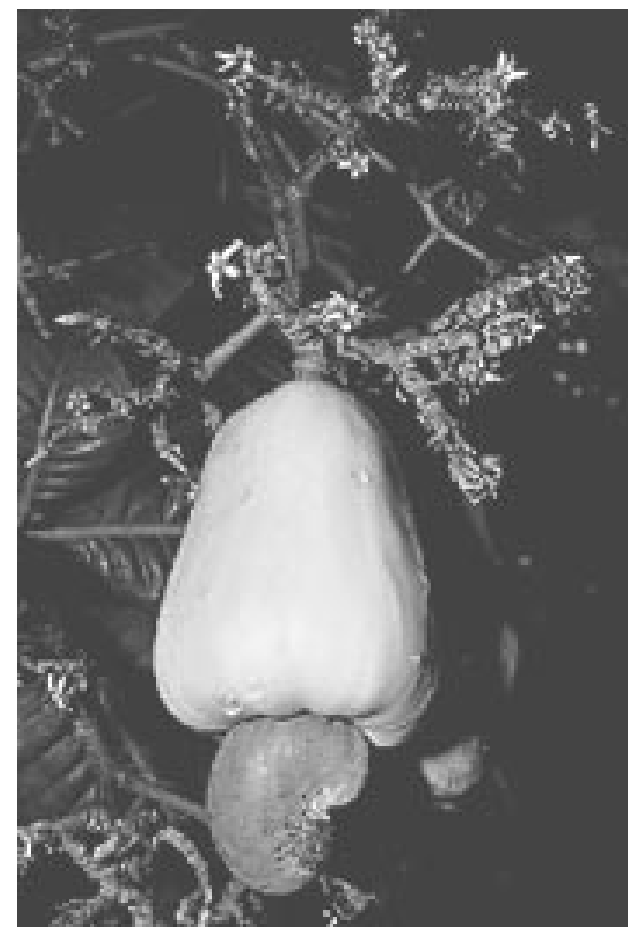

Tableau II.

Rendements annuels moyens en noix de cajou des principaux producteurs mondiaux en 1999 [7], présentés par ordre décroissant.

\begin{tabular}{lc} 
Pays & Rendement $\left(\mathrm{kg} \times \mathrm{ha}^{-1}\right)$ \\
\hline Tanzanie & 1247 \\
Mozambique & 786 \\
Nigéria & 633 \\
Inde & 611 \\
Guinée-Bissau & 400 \\
Côte d'Ivoire & 295 \\
Indonésie & 291 \\
Vietnam & 217
\end{tabular}

\subsection{Composition chimique de l'amande}

La composition moyenne de l'amande (tableau III) peut varier considérablement 


\begin{tabular}{|c|c|}
\hline Composé analysé & $\begin{array}{c}\text { Teneur } \\
\left(\mathrm{g} \times 100 \mathrm{~g}^{-1}\right. \\
\text { matière fraîche })\end{array}$ \\
\hline Humidité & 6,9 \\
\hline Protéines brutes & 21,5 \\
\hline Fibres brutes & 1,1 \\
\hline Lipides bruts & 46,1 \\
\hline Cendres & 3,4 \\
\hline Extraits non azotés (glucides) & 27,9 \\
\hline Calcium & 0,2 \\
\hline Phosphore & 0,7 \\
\hline
\end{tabular}

en fonction de la variété. L'amande de cajou se caractérise par sa richesse en acides gras essentiels, notamment en acides linoléique et oléique qui composent $20 \%$ et $60 \%$ de la matière grasse [4]. Elle est également riche en protéines et possède une composition en acides aminés semblable à celle de la graine de soja [2]. Dans certains cas, elle peut cependant présenter une déficience en isoleucine $[2,5]$. Les vitamines $P P$, $\mathrm{E}$ et $\mathrm{B} 1 \mathrm{y}$ sont présentes à des teneurs intéressantes soit, respectivement, 3,68, 2,10 et 0,56 mg pour $100 \mathrm{~g}$ d'amande [4].

\subsection{Transformation de la noix}

Depuis 1970, le traitement industriel de la noix de cajou tend vers une mécanisation complète de la ligne de production. Cependant, des difficultés importantes se présentent, liées à la disparité de la taille des noix et à la nature cassante des amandes.

En Inde, il existe une longue tradition de traitement manuel de la noix de cajou. Encore aujourd'hui, le savoir-faire indien conduit à des rendements en amandes entières inégalés. Pour cette raison, de nombreux autres pays producteurs ont longtemps exporté leurs noix entières en Inde. Face à d'autres contraintes socio-économiques, les producteurs brésiliens et africains ont généralement adopté des systèmes industriels plus mécanisés.

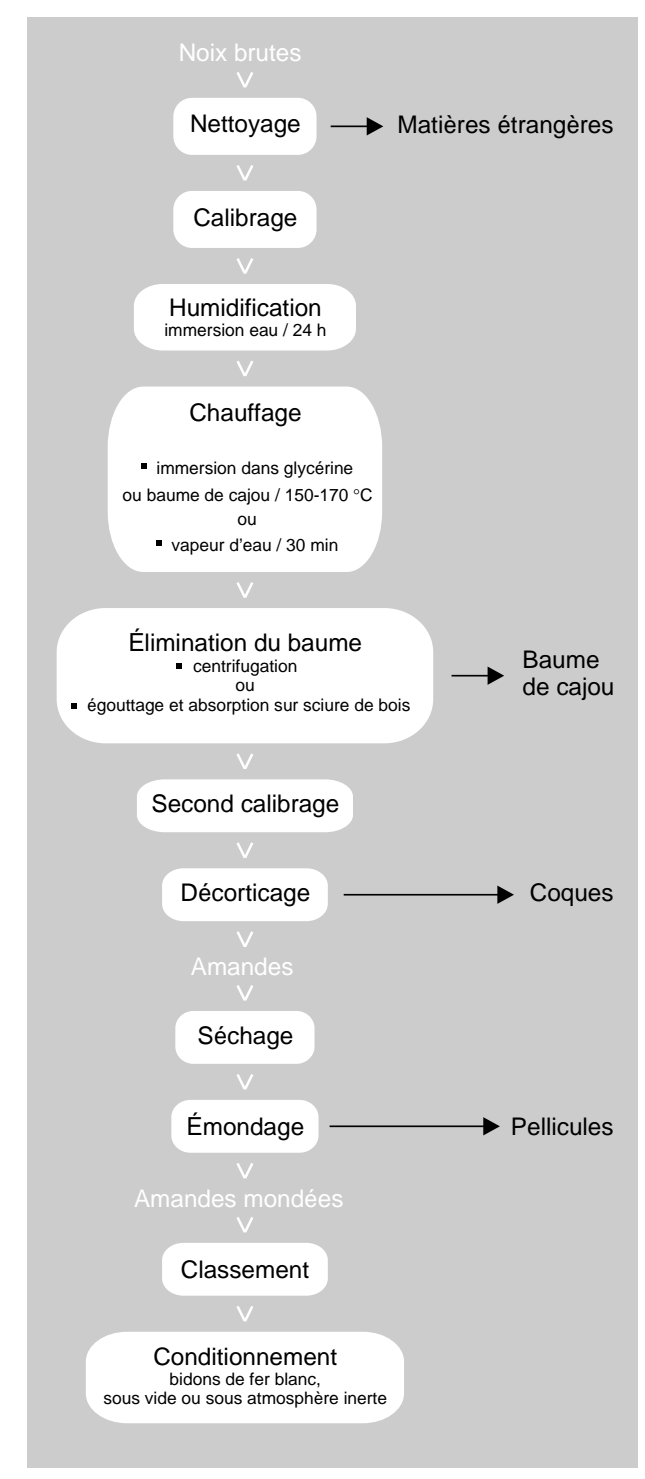

Figure 2.

Schéma type de la transformation industrielle de la noix de cajou [4].
La compétitivité des noix indiennes sur le marché international nécessite en effet une forte productivité pour être concurrentielle [2].

Le schéma général du procédé peut être divisé en quatre grandes étapes : les techniques préparatoires, le décorticage, l'émondage et le conditionnement (figure 2).

\subsubsection{Techniques préparatoires}

Les techniques préparatoires visent à fragiliser les coques avant le décorticage proprement dit. Dans les procédés de type 
artisanal, les noix subissent une torréfaction soit en récipient ouvert à l'air libre, soit dans un tambour rotatif.

Les procédés industriels permettent d'éviter les surchauffes liées au procédé traditionnel, qui nuisent à la qualité des amandes. Une réhumidification préalable des noix de 15 à $45 \%$ selon les méthodes est utilisée pour fragiliser la coque et rendre le traitement thermique plus efficace [5]. Celui-ci est réalisé par injection directe de vapeur ou par trempage dans un bain huileux. Ce bain peut être constitué de baume de cajou ; il a alors l'avantage de permettre la récupération du baume exsudé des noix portées à hautes températures. Le chauffage à la vapeur d'eau est cependant moins onéreux et permet d'éviter de soumettre le baume de cajou à des températures trop élevées. En effet, au-dessus de $150{ }^{\circ} \mathrm{C}$, le baume se polymérise et le rendement d'extraction est considérablement abaissé [4]. Afin d'éviter le contact entre l'amande et le baume qui conduit à la formation de taches sur l'amande, le baume est ensuite séparé des noix. Cette opération peut être réalisée soit par centrifugation - il est alors possible de le récupérer - soit par égouttage, puis absorption sur de la sciure de bois.

\subsubsection{Décorticage et émondage}

Selon les pays, le décorticage manuel permet de traiter de 2 à 7 noix par minute et par opérateur. Dans ce cas, les risques de brûlures occasionnées par le baume de cajou présent dans la coque de la noix nécessitent impérativement le port de gants de protection.

En utilisant un décorticage mécanique, la productivité est environ multipliée par 5 [5]. Les différents procédés de concassage sont basés sur trois principes : la percussion, le cisaillement (principe du procédé Oltremare) ou le fraisage. La séparation coque/amande est ensuite obtenue en utilisant un procédé pneumatique classique. Selon les procédés, les rendements varient de 60 à 83 \% d'amandes entières [4].

L'émondage consiste à séparer l'amande de la pellicule qui la recouvre, la testa. Après séchage au soleil ou à l'air chaud dans un séchoir, la séparation est réalisée totalement manuellement, à l'aide de couteaux ou encore par brossage doux sous aspiration. Les rendements sont de l'ordre de $70 \%$ et une finition manuelle est le plus souvent nécessaire [4]. Durant cette étape, un noircissement des amandes qui serait dû la complexation des tanins et du fer peut être observé. Un lavage acide permet alors d'y remédier [4].

\subsubsection{Conditionnement}

Après le classement, généralement effectué à la main, les amandes sont le plus souvent conditionnées dans des bidons de fer blanc soudés de 25 livres (11,35 kg). Le taux d'humidité doit être inférieur à $9 \%$ et, de préférence, compris entre 4 et $6 \%$ pour à la fois limiter la fragilisation des amandes trop sèches et éviter les contaminations fongiques [5]. Il est aussi possible d'utiliser un conditionnement sous vide ou sous gaz neutres $\left(\mathrm{N}_{2}\right.$ ou $\left.\mathrm{CO}_{2}\right)$ pour le conditionnement des amandes. Les amandes conditionnées sous vide peuvent ainsi être conservées 1 an, celles conditionnées sous $\mathrm{CO}_{2}, 2$ ans.

\subsection{Marchés de la noix}

L'évaluation des marchés de la noix de cajou est relativement difficile. En effet, les données économiques concernant ce produit sont rares. De plus, les marchés sont très spéculatifs et peu transparents.

Selon la FAO, en 1999, les principaux producteurs de noix de cajou étaient l'Inde, le Nigéria, le Brésil et la Tanzanie (figure 3). L'Afrique semble redevenir productrice de noix de cajou après une période de difficultés financières et politiques dans les pays concernés ; en effet, avant les années 1980, l'Afrique produisait plus de $40 \%$ de la production mondiale de noix de cajou. L'Inde et le Brésil ont, quant à eux, une longue tradition de production et de consommation : pendant la période de 1985 à 1989, l'Inde produisait $40 \%$ de la production mondiale et le Brésil, 35 \% [2]. En fait, ces deux importants protagonistes sur le marché mondial de la noix possèdent des périodes de récolte décalées de quelques mois et des marchés différents, mais, le plus souvent, ils sont en 


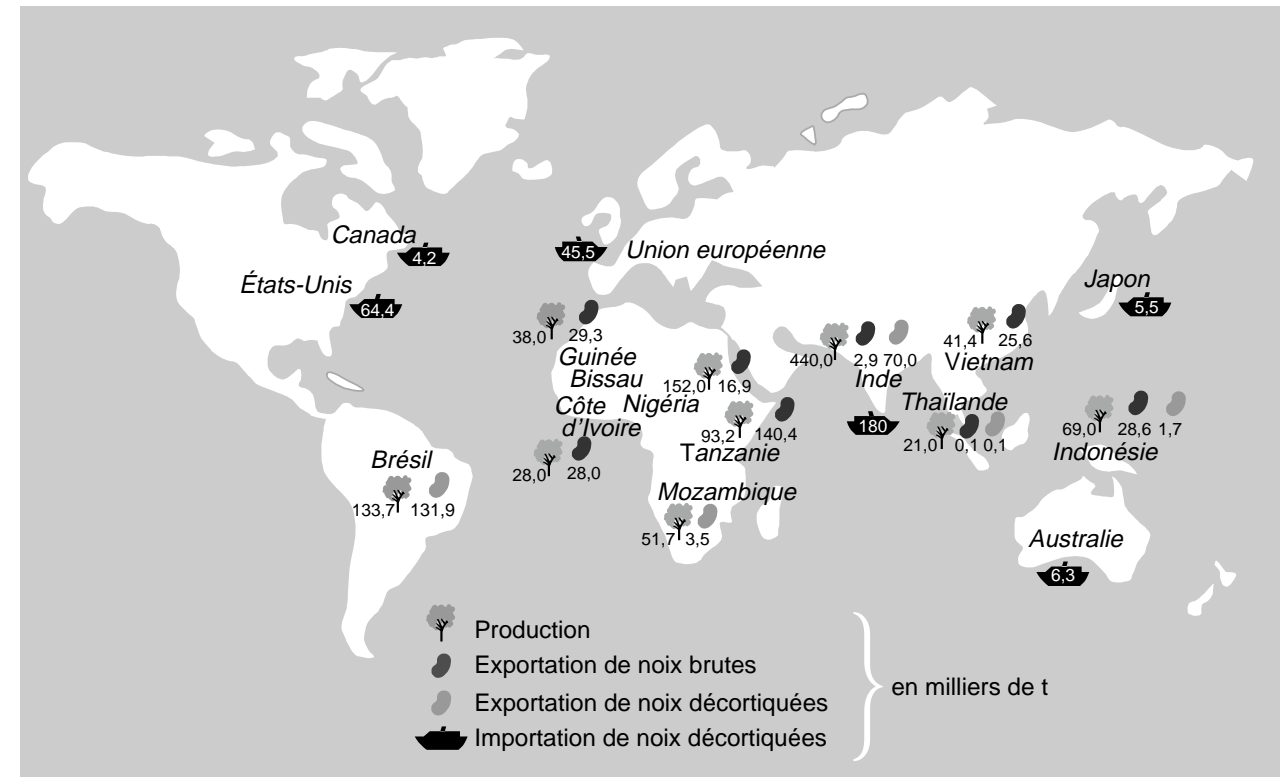

concurrence. Cela est d'autant plus vrai que la production indienne, qui trouvait un débouché dans l'ex-URSS, est désormais exportée vers les États-Unis, client traditionnel du Brésil.

Alors que les zones de production se trouvent dans des régions intertropicales, la demande en noix de cajou est essentiellement localisée dans les régions tempérées qui possèdent un fort pouvoir d'achat. Ainsi, en 1990, les principaux importateurs étaient les États-Unis, l'ex-URSS, le Royaume-Uni, le Canada, le Japon, les PaysBas, l'Allemagne et l'Australie avec $90500 \mathrm{t}$ importées par les dix principaux importateurs [2]. Le marché mondial est fortement influencé par la demande et par la variabilité des volumes de production. Entre 1981 et 1991, les prix moyens import annuels des noix de cajou de qualité WW320 (= White Wholes à 320 amandes par livre) ont fluctué entre environ 4 \$US et 7 \$US par kg [2]. En comparaison avec les autres noix comestibles, les prix de la noix de cajou restent néanmoins relativement élevés (tableau IV).

Outre les flux sud-nord, le marché de la noix de cajou se caractérise par d'importants flux sud-sud d'échanges de noix brutes (figure 4). Si certains pays comme la Tanzanie ou la Côte d'Ivoire sont exclusivement producteurs et exportent la quasitotalité de leurs noix non transformées,

\section{Figure 3.}

Principaux pays producteurs et leurs exportations, et principaux pays importateurs de noix de cajou en 1998 [7].

\section{Tableau IV.}

Exemple de prix moyens import de différentes noix comestibles en $\$ \mathrm{US} \times \mathrm{kg}^{-1}$ [2].

\begin{tabular}{|c|c|c|c|c|c|c|}
\hline \multirow{2}{*}{$\begin{array}{l}\text { Période } \\
\text { (1 } \text { (er }^{\text {semestre }} \\
\text { de l'année) }\end{array}$} & \multicolumn{6}{|c|}{ Type de noix / qualité } \\
\hline & $\begin{array}{l}\text { Noix de cajou / } \\
\text { WW320 }\end{array}$ & $\begin{array}{l}\text { Amande / } \\
\text { NPSSR }^{2}\end{array}$ & $\begin{array}{c}\text { Noisettes / } \\
\text { Turkish levant }\end{array}$ & $\begin{array}{c}\text { Noix } \\
\text { d'Amazonie / } \\
\text { demi-amande }\end{array}$ & $\begin{array}{l}\text { Cacahuète / } \\
\text { US Runner }\end{array}$ & $\begin{array}{l}\text { Noix de pécan / } \\
\text { Mammoth halves }\end{array}$ \\
\hline 1981 & 7,06 & 3,61 & 3,46 & 2,29 & 1,77 & - \\
\hline 1986 & 6,69 & 3,69 & 3,26 & 1,72 & 0,77 & 6,63 \\
\hline 1991 & 6,09 & 3,10 & 2,76 & 3,00 & 1,46 & 9,90 \\
\hline
\end{tabular}

1 WW320: White Wholes à 320 amandes par livre.

2 NPSSR : Non Pareil Select Sheller Run. 


\section{Figure 4.}

Répartition des importations en noix brutes à l'échelle mondiale, pour l'année 1998 [7].
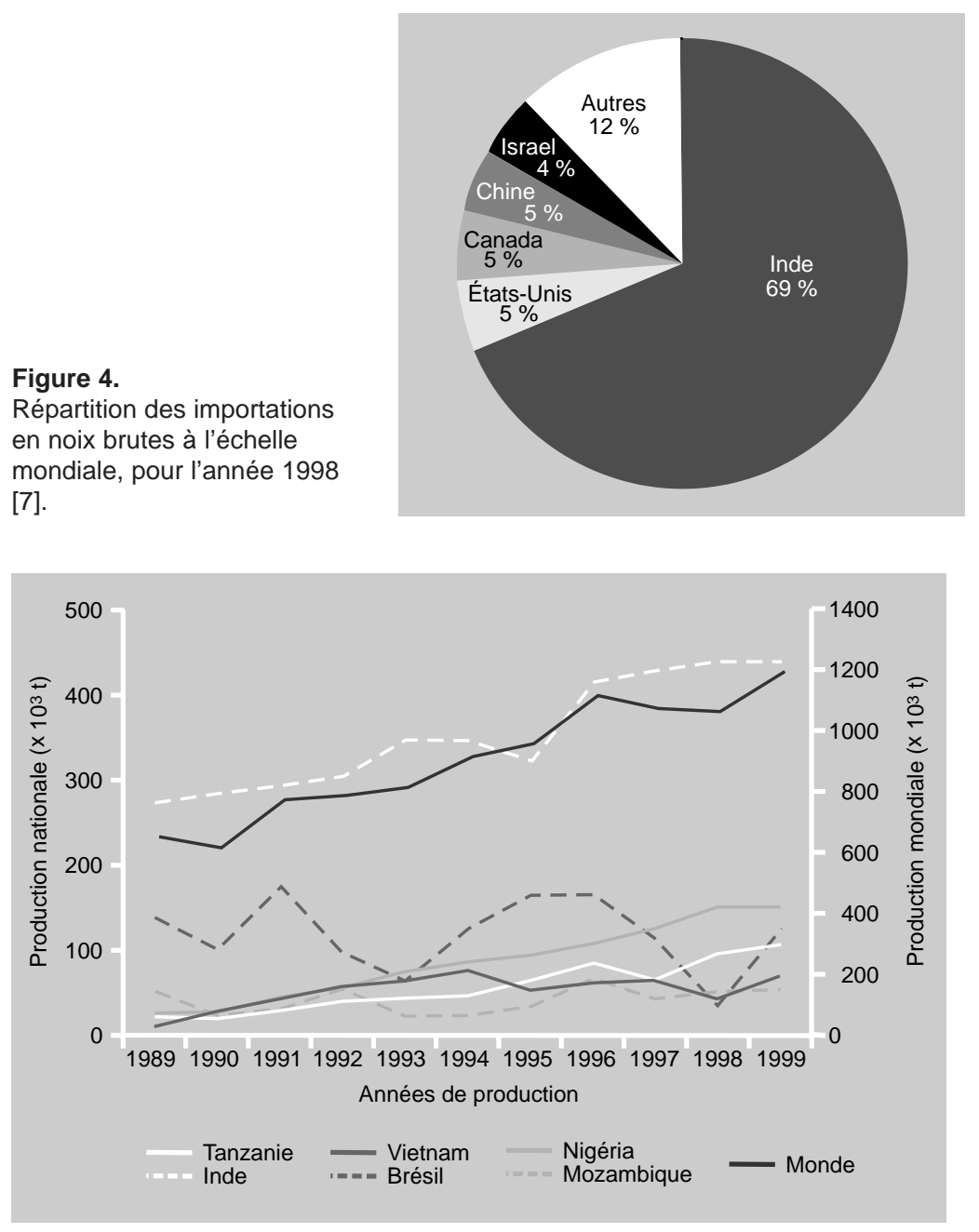

Figure 5.

Évolution de la production de noix de cajou pour les principaux pays producteurs, entre 1989 et 1999 [7]. d'autres, comme le Brésil, produisent et transforment les noix de cajou, mais uniquement celles issues de leur propre production (figure 3). Entre ces deux situations extrêmes, certains pays producteurs comme l'Indonésie exportent la majorité de leur production en noix brutes mais en transforment une partie. Dans le contexte actuel, l'Inde correspond à un schéma très particulier. En plus de transformer sa propre production déjà très conséquente, ce pays importe de 25 à $40 \%$ des noix qu'il transforme. Mettant à profit le décalage des saisons de production pour alimenter de façon continue les usines de transformation, l'Inde importe ainsi $70 \%$ des noix brutes mondiales (figure 4). Ce pays peut donc être considéré comme la véritable plaque tournante du marché de la noix de cajou et oriente fortement les prix internationaux. Les États-Unis, qui sont presque exclusivement transformateurs et importateurs de noix déjà décortiquées et qui ne produisent pratiquement pas de noix de cajou brutes, représentent un cas particulier.

L'analyse de l'évolution du marché depuis 1989 (figure 5), montre que la tendance globale est à la hausse. En effet, la production mondiale de noix de cajou a presque doublé en 10 ans. La production de pays comme l'Inde, le Nigéria, la Tanzanie et le Vietnam a subi une croissance relativement régulière. Bien que, durant ces dix dernières années, l'Inde soit restée de loin le premier producteur mondial, les productions du Nigéria et du Vietnam ont été multipliées respectivement par six et par huit, de 1989 à 1998. Le Mozambique, en proie à d'importants troubles politiques, n'enregistre pas cette croissance et sa production stagne. Quant au Brésil, il possède la production la plus irrégulière. Ayant bénéficié d'une politique de subventions d'État pendant les années 1970 et 1980, ce pays a été sans conteste le deuxième producteur mondial jusqu'en 1992. Depuis cette date, de graves problèmes de sécheresse dans le Nordeste, principale région productrice au Brésil, expliquent les chutes brutales de la production nationale en 1993 et 1998. La production brésilienne est désormais comparable à celle des autres grands producteurs et elle est inférieure à celle du Nigéria depuis trois ans déjà.

Notons enfin qu'au cours de ces dernières années, de nombreux projets de relance de la production de noix de cajou ont été mis en place, notamment en Afrique, dans l'océan Indien et en Asie du Sud-Est. Ces actions devraient se traduire à court terme par des évolutions significatives du marché mondial de la noix.

\subsection{Normes à l'exportation}

Afin de garantir aux acheteurs une qualité constante, la plupart des producteurs et importateurs se réfèrent à des normes de qualité définies au sein d'un même marché. La norme ISO 6477 de février 1988 réunit les deux types de classifications utilisées 
par l'Inde et les pays africains d'une part et le Brésil d'autre part, en donnant un critère unique pour évaluer la qualité des lots d'amandes [2].

Cette classification est basée sur le fait que l'amande est entière ou pas, sur son aspect (blanche, colorée ou tachée) et sur la taille des amandes ou des morceaux. Pour les amandes entières, la taille est évaluée par le nombre d'amandes contenues dans une livre. En revanche, pour les amandes brisées, une distinction est faite entre les moitiés, les bouts, les morceaux, les petits morceaux et les fragments. Pour chacune de ces catégories, un taux de $5 \%$ d'amandes appartenant à une catégorie inférieure est toléré.

À titre d'exemple, les amandes entières blanches correspondent à la catégorie WW (White Wholes). Ces amandes doivent être réniformes et ne présenter aucune forme de contamination, ni dommage causé par les insectes, ni moisissures, ni goût de rance, ni résidus de pellicule ou de matières étrangères. Elles doivent être blanches, à tendance grise ou ivoire. Dans cette catégorie, les amandes bosselées sont autorisées à condition que leurs formes soient homogènes. Le classement indo-africain précise les codes WW180, WW210, WW240, WW320, WW400, WW450 et WW500 pour lesquelles le chiffre indique le nombre d'amandes dans une livre (indication de taille). Le classement brésilien ne spécifie, quant à lui, que trois catégories de tailles : SLW1 (de 160 à 180 amandes par livre), LW1 (de 180 à 210 amandes par livre) et W1 (pour les amandes plus petites). L'humidité maximale tolérée est de $5 \%$.

\section{Le faux fruit de l'anacardier: la pomme de cajou}

\subsection{Description et composition}

Après le développement de la noix, son pédoncule s'hypertrophie et change de couleur pour donner un faux fruit connu sous le nom de pomme de cajou. À maturité, cette pomme de cajou est de couleur rouge,

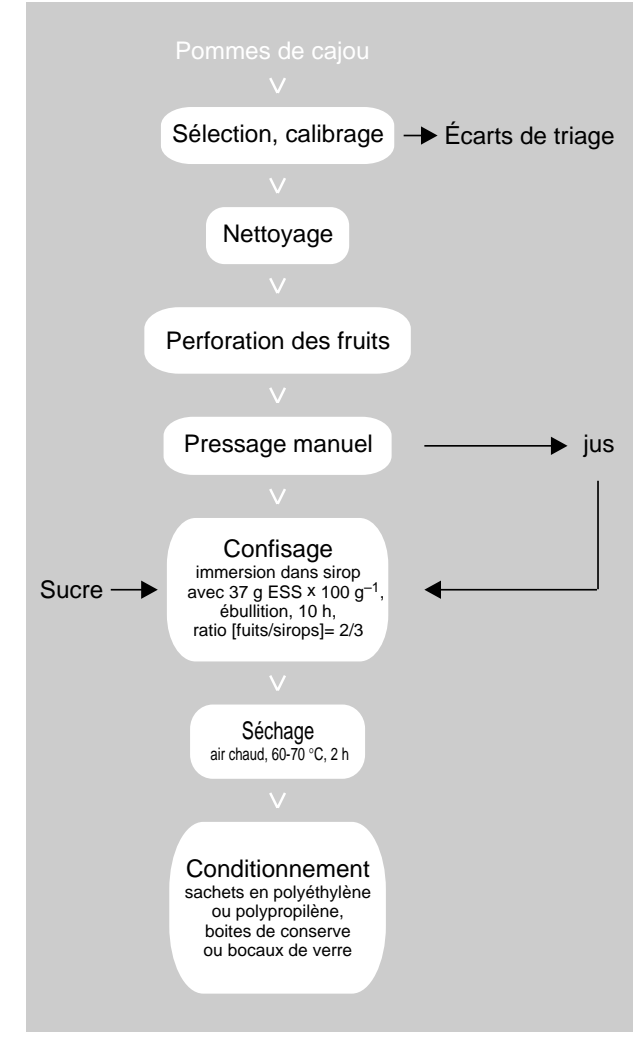

Figure 6.

Exemple de schéma d'élaboration d'un produit sucré traditionnel à base de pomme de cajou (faux fruit de l'anacardier) : le « caju-ameixa » brésilien [1]. jaune ou orangée. En revanche, quelle que soit la couleur de la fine pellicule cireuse qui constitue la peau du fruit, sa chair est jaune. La forme de ce faux fruit est ronde ou piriforme (sa dénomination, Anacardium, signifie «en forme de cœur $\gg$ et il peut atteindre $10 \mathrm{~cm}$ de long et $5 \mathrm{~cm}$ de large [5]. Le poids d'une pomme de cajou est généralement compris entre 70 et $90 \mathrm{~g}$ [8].

La pomme mûre possède une odeur très particulière qui peut surprendre le néophyte [9]. D'après la littérature, l'arôme de la pomme de cajou est relativement mal caractérisé. Sa teneur en composés d'arôme est faible par rapport aux autres fruits tropicaux. Une étude réalisée sur des pommes cajou vénézuéliennes a montré que le car3-ène représentait environ $25 \%$ de la quantité totale des composés aromatiques de la pomme [10]. Ce composé d'arôme, qui est également présent dans la mangue, est responsable d'une odeur fruitée douceâtre. Néanmoins, aucune étude qualitative sur les seuils de détection des différents 


\begin{tabular}{|c|c|}
\hline Composé analysé & Teneur pour $100 \mathrm{~g}$ de matière fraîche \\
\hline $\begin{array}{l}\text { Extrait sec soluble } \\
\text { pH } \\
\text { Acidité titrable (acide malique) } \\
\text { Sucres réducteurs } \\
\text { Fibres alimentaires } \\
\text { Tanins } \\
\text { Protéines } \\
\text { Vitamine C }\end{array}$ & $\begin{array}{l}10 \text { à } 12 \mathrm{~g} \\
4,2 \text { à } 4,4 \\
0,33 \text { à } 0,59 \mathrm{~g} \\
7,8 \text { à } 8,6 \mathrm{~g} \\
2,6 \text { à } 3,1 \mathrm{~g} \\
0,29 \text { à } 0,38 \mathrm{~g} \\
0,7 \text { à } 0,8 \mathrm{~g} \\
200 \text { à } 300 \mathrm{mg}\end{array}$ \\
\hline $\begin{array}{l}\text { Sels minéraux : } \\
\text { Calcium } \\
\text { Fer } \\
\text { Phosphore }\end{array}$ & $\begin{array}{c}10 \text { à } 40 \mathrm{mg} \\
0,4 \text { à } 3,0 \mathrm{mg} \\
10 \text { à } 30 \mathrm{mg}\end{array}$ \\
\hline
\end{tabular}

composés d'arômes présents et leur impact sensoriel sur l'arôme de la pomme de cajou n'est disponible actuellement.

De la pomme de cajou peut être extrait $85 \%$ de jus. Ce jus contient environ $10 \%$ de sucres qui sont en majorité des sucres réducteurs (tableau $\mathrm{V}$ ). La pomme contient également une proportion relativement importante de composés polyphénoliques (tannins) dont certains sont responsables de son astringence caractéristique. L'intensité du goût sucré et de l'astringence est assez variable en fonction de la variété et du stade de maturité. La teneur en vitamine $\mathrm{C}$ de la pomme de cajou est comprise entre 200 et $300 \mathrm{mg} \times 100 \mathrm{~g}^{-1}$ de matière fraîche, soit environ 5 fois celle de l'orange. Sa teneur importante en fibres alimentaires est liée à sa nature biologique. En effet, le pédoncule hypertrophié est constitué de fibres vasculaires longues et épaisses, agencées en une vingtaine de faisceaux reliant le point d'insertion sur la branche et celui de la noix. Au voisinage de ces faisceaux, les activités enzymatiques semblent être particulièrement importantes [11].

\subsection{Récolte et conservation}

La très faible durée de conservation de la pomme de cajou à température ambiante, entre $24 \mathrm{~h}$ et $48 \mathrm{~h}$ maximum, est le principal facteur limitant de la qualité, tant sur le plan de la vente en frais que sur celui de la transformation. La détérioration de la qualité du produit est très rapide du fait des caractéristiques physiologiques de cet organe végétal. Ce fruit non climactérique est en effet caractérisé par une intensité respiratoire particulièrementélevée, supérieure à la plupart des fruits tropicaux, équivalente à $74-76 \mathrm{~mL} \mathrm{O}_{2} \times \mathrm{kg}^{-1} \times \mathrm{h}^{-1}$ et $62-72 \mathrm{~mL}$ $\mathrm{CO}_{2} \times \mathrm{kg}^{-1} \times \mathrm{h}^{-1}$ [1]. De plus, sa forte teneur en eau et son pH voisin de 4,2 sont assez favorables à la croissance microbienne.

Après un traitement permettant de diminuer la charge microbienne de surface (bain d'acide citrique et bisulfite, d'acide sorbique ou d'eau chlorée), les conditions optimales de conservation correspondent à une température basse (jusqu'à 0 à $2^{\circ} \mathrm{C}$ ) et une humidité relative élevée de l’ordre de 90 \% [8]. À cause de leur grande fragilité, les pommes de cajou doivent être récoltées immédiatement après l'absission ou bien être cueillies à la main. Cela impose donc que la récolte ait lieu fréquemment. Notons cependant que le tonnage récolté à l'hectare pour la pomme de cajou est de 5 à 9 fois celui de la noix.

\subsection{Voies de valorisation}

La pomme de cajou, malgré sa grande fragilité, possède de nombreux atouts. En plus de sa qualité nutritionnelle, la pomme présente des intérêts technologiques : la partie comestible du fruit (entre $85 \%$ et $100 \%$ ) est supérieure à celle d'autres fruits tropicaux traditionnels et sa chair juteuse et sucrée est exempte de pépin ou de noyau. De plus, des volumes très importants sont disponibles si l'on considère les pommes issues de l'exploitation de la noix de cajou. En considérant que la masse de la pomme est cinq à neuf fois celle de la noix, il a été possible d'estimer la production totale de pommes de cajou dans le monde, en 1999, à entre 6 et $10 \mathrm{Mt}$. La valorisation de ce faux fruit, souvent considéré comme un sousproduit de l'exploitation de la noix, représente donc un enjeu économique considérable. Notons néanmoins que, pour l'année 1999, seuls le Brésil et Madagascar ont été recensés comme producteurs de pommes de cajou avec 1,5 Mt et 0,069 Mt, respectivement [7]. 
Il existe une large gamme de produits à base de pomme de cajou : les jus et pulpes, les produits déshydratés, les pâtes de fruits, les compotes, les chutneys et les boissons fermentées et distillées. Actuellement, seuls le jus et la pulpe de pomme de cajou sont produits à grande échelle.

\subsubsection{Fruit frais}

Les marchés du frais restent exclusivement régionaux. Le principal obstacle à leur développement est évidemment la faible durée de conservation de la pomme de cajou. Lors du stockage réfrigéré, certains auteurs ont mentionné des problèmes de «maladies $\mathrm{du}$ froid » qui limitent considérablement l'intérêt de ce mode de conservation pour allonger de manière intéressante la durée de vie commerciale du produit [1]. À l'heure actuelle, les traitements après-récolte mis en œuvre permettent d'atteindre des durées de conservation comprises entre 1 et 3 semaines. Des études sur l'utilisation d'atmosphères contrôlées ou modifiées associées à un emballage adéquat sont actuellement en cours et devraient conduire à une amélioration significative de ces résultats [1].

\subsubsection{Jus de fruit}

L'extraction du jus est généralement réalisée à l'aide d'un pressoir ou d'un épulpeur centrifuge. Ces deux techniques peuvent éventuellement être couplées pour augmenter le rendement d'extraction [4]. Le rendement dépend fortement de l'état de maturité des fruits et de l'unité de transformation elle-même. À titre d'exemple, un rendement compris entre $50 \%$ et $65 \%$ peut être obtenu avec une presse continue à vis [12]. Dans tous les cas, les unités d'extraction doivent impérativement être localisées au voisinage immédiat des zones de production, compte-tenu de la faible durée de conservation des fruits.

Après extraction, les jus sont le plus souvent clarifiés après avoir subi un traitement pour réduire leur teneur en tannins. En effet, ces composés phénoliques, en plus de leur astringence, contribuent à l'instabilité des jus en favorisant l'apparition de dépôts. Différentes techniques ont été mises au point pour diminuer la teneur en tannins des jus. La plus utilisée est un collage à la gélatine suivi d'une filtration qui permet de diminuer la teneur en tannins de 0,3 \% à 0,03\%. Cette méthode conduit à des pertes en acide ascorbique relativement modérées, de l'ordre de $17 \%$ [5]. Un mélange avec des fruits riches en pectines (goyaves, grenadilles) pourrait aussi être envisagé pour diminuer la teneur en tannins [12]. La couleur et la flaveur des jus clarifiés sont généralement jugées meilleures que celles des jus non clarifiés. De plus, la clarification permet de diminuer significativement la viscosité des jus, aspect particulièrement important si une concentration est envisagée [5]. Après conditionnement, la stabilisation du jus est classiquement obtenue par pasteurisation. L'ajout de métabisulfite de sodium $\left(1 \mathrm{~g} \times \mathrm{kg}^{-1}\right)$ réduit les brunissements et permet une bonne conservation à température ambiante ( 24 à $30^{\circ} \mathrm{C}$ ) pendant 16 semaines [5].

Cette voie de valorisation est très développée au Brésil. En effet, environ 50000 t de jus de cajou ont été produites dans ce pays en 1998, ce qui représente $50 \%$ de la production nationale brésilienne de jus de fruit, hors jus d'orange. La croissance de ce secteur y est estimée à 10-15\% par an [8].

\subsubsection{Autres transformations traditionnelles}

\subsubsection{Confiture de cajou}

$\mathrm{Au}$ XVIII siècle, la confiture de cajou était assez populaire en France, puis son utilisation a été diffusée au reste de l'Europe [2]. En Inde, des pommes très mûres sont utilisées pour réaliser ces préparations [4, 12] : elles sont d'abord immergées 3 jours dans une solution saline à $2 \%$ afin de diminuer leur astringence, puis abondamment rincées, pelées et blanchies 10 à $15 \mathrm{~min}$ à la vapeur. La confiture est obtenue après cuisson pendant 2 à $3 \mathrm{~h}$ à $105^{\circ} \mathrm{C}$ du mélange pomme/sucre (50:50). Une acidification en fin de cuisson est recommandée (jus de citron ou acide citrique). 


\subsubsection{Autres préparations sucrées}

De nombreux autres produits sucrés à base de pomme de cajou sont traditionnellement élaborés au Mozambique, en Inde et au Brésil. Dans ce dernier pays, on citera par exemple le "caju-ameixa ", pâte noire de texture souple semblable à la crème de pruneaux (figure 6). En Inde, la préparation de pommes confites peut également être mentionnée : les fruits sont tout d'abord immergés dans des solutions salées de plus en plus concentrées (2 à $10 \%$ ). Après cette étape qui dure environ une semaine et addition éventuelle de $0,5 \%$ de métabisulfite de potassium, les fruits sont rincés, pelés et triés. Ils sont ensuite progressivement confits dans des sirops de concentration croissante, puis glacés par trempage rapide dans un sirop bouillant à $70 \mathrm{~g} \mathrm{ESS} \times 100 \mathrm{~g}^{-1}$ [12].

\subsubsection{Chutneys}

Les chutneys, produits traditionnels indiens très appréciés par les Anglo-saxons, sont des pâtes épicées sucrées-salées qui sont consommées comme condiments [12]. Après saumurage ( $2 \%$ de $\mathrm{NaCl})$, lavage et blanchiment à la vapeur (5 à $7 \mathrm{~min}$ ), les pommes cajou sont pelées puis tranchées. Le produit final est obtenu après cuisson des morceaux de pommes avec du sucre $\left(1,2 \mathrm{~kg} \times \mathrm{kg}^{-1}\right)$, $\mathrm{du}$ sel, du vinaigre, des oignons frits, du gingembre et diverses épices.

Figure 7.

Structure des principaux composés phénoliques caractéristiques du baume de cajou extrait de la coque de la noix de cajou [2].

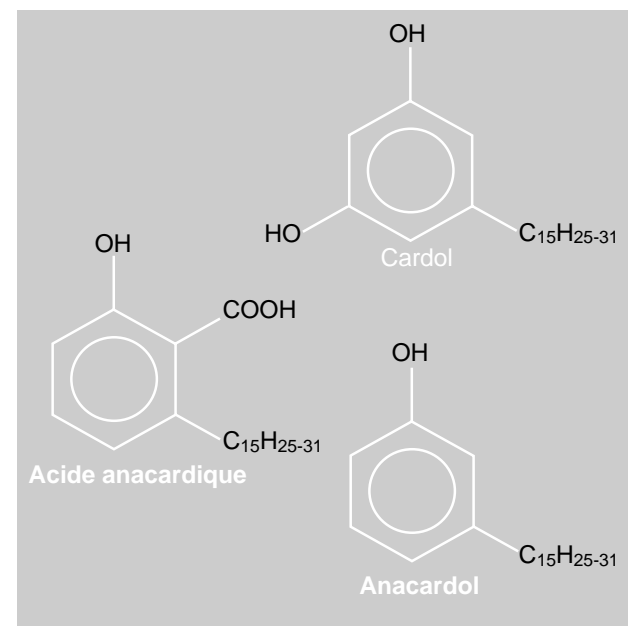

\section{Autres productions de l'anacardier}

La coque qui correspond à $50-55 \% d u$ poids de la noix est constituée d'alvéoles qui contiennent un liquide acide. Cette résine phénolique vésicante et toxique est appelée baume de cajou [4]. Le baume de cajou ou «cashew nut shell liquid »(CNSL) est un liquide foncé et très visqueux constitué d'environ $14 \%$ de cardol et de $80 \%$ d'acide anacardique (figure 7). Lorsque le produit est chauffé, la composition chimique du baume se modifie [2]. L'acide anacardique subit notamment une décarboxylation pour donner du cardanol, composé qui est utilisé dans l'industrie des peintures. Le baume de cajou est séparé par centrifugation des coques brisées. Cette opération permet de récupérer environ $90 \%$ du produit et peut être complétée par une extraction par solvant [2]. Le baume représente une source importante et économique de composés phénoliques naturels qui peuvent être utilisés dans la fabrication d'éléments de friction pour l'industrie automobile, dans l'industrie des revêtements spéciaux (vernis, matières plastiques) et pour la formulation d'insecticides. C'est à partir du milieu des années 1970 que le baume, sous-produit de l'industrie de la noix de cajou, a suscité un intérêt industriel. En 1990, le principal producteur de baume a été le Brésil avec environ $30000 \mathrm{t}$, puis l'Inde et le Mozambique [2]. Les principaux importateurs de baume sont les États-Unis, le Royaume-Uni et le Japon.

Accessoirement, d'autres produits sont valorisés à partir de l'industrie de la noix. À partir des débris d'amandes émondées, il est possible d'obtenir du beurre de cajou, produit similaire au beurre d'arachide, ou de l'huile d'amande de cajou. Cette huile est obtenue avec un taux d'extraction de $37 \%$ à $57 \%$, par pression des débris d'amandes [12]. Elle est fluide, jaune pâle, inodore et d'excellente qualité nutritionnelle. En fait, sa composition en acides gras fait d'elle une huile intermédiaire entre l'huile d'arachide et l'huile d'olive [2]. Les teneurs en acides oléique et stéarique y sont importantes. Le rapport [acides saturés/acides insaturés] est de 4,1, la valeur idéale préconisée par les 
nutritionnistes étant de 5. La composition en acide linoléique $(17,1 \%)$ est également intéressante sur le plan nutritionnel.

Les tourteaux issus du pressage sont riches en protéines et en glucides. Après broyage, ils peuvent être utilisés pour l'alimentation du bétail. Les pellicules (testa) sont riches en protéines et servent, en Inde, d'alimentation pour les volailles à condition de ne pas contenir trop de baume. Après le grillage et le décorticage des noix, environ $30 \mathrm{~kg}$ de pellicules sont récupérées par tonne de noix [12]. Par ailleurs, les coques issues du décorticage des noix se prêtent bien à la fabrication de panneaux agglomérés. En revanche, les coques trop finement broyées pour extraire le baume sont difficilement valorisables.

\section{Conclusion}

La noix de cajou, le fruit au sens botanique du terme, est le produit de l'anacardier le plus connu et le mieux valorisé. L'amande manufacturée possède en effet une valeur ajoutée importante. Elle est bien intégrée aux marchés internationaux. Les exportations de noix constituent une source de revenu non négligeable pour les pays producteurs.

La pomme de cajou est, en revanche, encore nettement sous-utilisée à l'échelle mondiale. Pourtant, avec un rapport massique [noix/pomme] compris entre $1 / 5$ et $1 / 9$, elle pourrait être plus rémunératrice pour le producteur que la noix. Sa valorisation se heurte néanmoins à deux principaux facteurs limitants : la difficulté de conserver la pomme en frais et son astringence. Ce dernier aspect freine considérablement l'acceptation du produit par des consommateurs non avertis et, donc, limite les marchés d'exportation. À cet égard, la sélection variétale devrait jouer un rôle essentiel dans l'avenir. Enfin, l'utilisation de technologies susceptibles de diminuer l'astringence de la matière première devrait permettre d'obtenir des produits à base de pomme de cajou de qualité gustative plus en adéquation avec la demande internationale (notamment produits déshydratés).
L'effort de recherche a été jusqu'ici essentiellement focalisé sur le produit à plus forte valeur ajoutée : la noix. Cependant, la qualité aromatique de la pomme de cajou, sa maturation, sa conservation, ainsi que la nature des composés phénoliques qu'elle contient, sont autant d'aspects qui sont encore aujourd'hui mal connus. Il paraît essentiel de les approfondir afin d'envisager le développement de ce produit.

\section{Références}

[1] Merabtine E., Valorisation de la pomme de cajou : différentes voies d'obtention de produits déshydratés pour une incorporation dans les produits laitiers, Mémoire de fin d'études d'ingénieur, École nationale supérieure des Industries alimentaires, Section Industries alimentaires régions chaudes (Ensia-Siarc), Montpellier, France, 1998.

[2] De Logu A.M., Haeusler G., The world cashew economy, Inchiostroblu, Bologne, Italy, 1994.

[3] Aogou S.A., L'anacardier, Anacardium occidentale, famille des anacardiacées, Le Flamboyant 38 (1996) 7-11.

[4] Dogo N.N., N'Guetta M., Neves E., L'anacardier, valorisation du faux fruit et du fruit, Dossier thématique, École nationale supérieure des Industries alimentaires, Section Industries alimentaires régions chaudes (Ensia-Siarc), Montpellier, France, 1999.

[5] Wardowski W.F., Ahrens M.J., Cashew Apple and Nut, in: Nagy S., Shaw P.E., Wardowski W.F. (Eds), Fruits of tropical and subtropical origin, Florida Sci. Source, Lake Alfred, USA, 1990.

[6] Crane J.H., Campbell C., Origin and distribution of tropical and subtropical fruits, in: Nagy S., Shaw P.E., Wardowski W.F. (Eds), Fruits of tropical and subtropical origin, Florida Sci. Source, Lake Alfred, USA, 1990.

[7] Anonyme, FAOSTAT, Banque de données de l'Organisation des Nations unies pour l'alimentation et l'agriculture, Adresse URL : www.fao.org, 2001.

[8] Reynes M., Valorisation de la pomme de cajou, Compte rendu de mission effectuée au Brésil 4-18 mai, Rapport interne, Centre de coopération international en recherche agronomique pour le Développement (Cirad), Montpellier, France, 1998. 
[9] Ohler J.G., Cashew, Kominklijk Instituut voor de Tropen, Amsterdam, Netherlands, 1979.

[10] Mac Leod A.J., de Troconis N.G., Volatile flavour components of cashew apple (Anacardium occidentale), Phytochemistry 21 (1982) 2527-2523.

[11] Lautié E., Valorisation de la pomme de cajou par déshydratation osmotique sous vide,
Mémoire de thèse de Master, École nationale supérieure des Industries alimentaires, Section Industries alimentaires régions chaudes (Ensia-Siarc), Montpellier, France, 2000.

[12] Haendler L., Duverneuil G., Note sur les possibilités de transformation des fruits et des faux fruits de l'anacardier, Fruits 25 (1970) 379-384.

\section{El anacardo y sus productos: características principales y medios de valorización.}

Resumen - Introducción. Procedente de Brasil, el anacardo está presente en muchos países de la zona intertropical. Este trabajo permite hacer una synthesis de los intereses económicos y las diferentes maneras de usar los productos de un árbol tan extenso. El anacardo. Anacardium occidentale (Anacardiacea) es un tipo de árbol bastante resistente, particularmente a los períodos de sequía, lo que le vuelve un árbol bien adaptado a muchas zonas tropicales. A pesar de su tolerancia a los ataques de parásitos, puede ser sensible a algunos insectos y algunos ataques de hongos como la antracnosis. Los rendimientos medios son aproximadamente de $600 \mathrm{~kg} \times \mathrm{ha}^{-1} \times \mathrm{anno}^{-1}$ de nuez y pueden ser mejorados gracias a la selección varietal. La nuez de anacardo. Es la verdadera fruta al sentido biológico. La almendra es rica en lípidos y su contenido en proteínas está aproximadamente de $20 \%$. Los principales productores del mundo en 1999, con una producción superior para cada un a 100000 t, son India, Nigeria, Brasil y Tanzania. Después de machacar el casco y de descascarar la almendra, este producto con alta valor adquirida está generalmente exportado. La manzana de anacardo. El pedunculo hipertrofiado de la nuez se vuelve, con la madurez, una fruta jugosa y azucarada. Tiene como características su astringencia y su contenido en vitamina $\mathrm{C}$ (200-300 mg $\left.\times 100 \mathrm{~g}^{-1}\right)$. La manzana de anacardo es poco usada excepto en India y en Brasil, como zumo de fruta por ejemplo. Otras producciones del anarcardo. El bálsamo de anacardo (CNSL) es una producción secundaria original: extraio del casco de la nuez, este líquido corrosivo compuesto principalmente de polyphenolicos se utiliza en la industria química. Conclusión. La cultura del anacardo con su diversidad de productos, particularmente con la nuez que está bien integrada a los mercados internacionales, parece poder constituir una fuente interesante de rentas para las zonas tropicales secas. No obstante, la manzana requiere ser desarrollada mejor en muchos lugares porque constituye, al momento, un subproducto de la industria del anacardo que tiene un potencial fuerte.

Anacardium occidentale L. / anacardo / composición química / procesamiento / mercados de productos básicos / mercados mundiales / usos / jugo de frutas / taninos 